\title{
Identification of the active substances and mechanisms of ginger for the treatment of colon cancer based on network pharmacology and molecular docking
}

Meng-Meng Zhang ${ }^{1}$, Dan Wang ${ }^{1}$, Feng Lu' ${ }^{1}$, Rong Zhao ${ }^{1}$, Xun Ye ${ }^{1}$, Lin $\mathrm{He}^{1}$, Li Ai ${ }^{2^{*}}$ (D) and Chun-Jie Wu ${ }^{1 *}$

\author{
* Correspondence: aili@cdutcm.edu. \\ cn; wucjcdtcm@163.com \\ ${ }^{2}$ School of Ethnic Medicine, \\ Chengdu University of Traditional \\ Chinese Medicine, No.1166 Liutai \\ Avenue, Chengdu 611137, P. R. \\ China \\ 'School of Pharmacy, Chengdu \\ University of Traditional Chinese \\ Medicine, No.1166 Liutai Avenue, \\ Chengdu 611137, P. R. China
}

\begin{abstract}
Background and objective: Colon cancer is occurring at an increasing rate and ginger (Zingiber officinale), as a commonly used herbal medicine, has been suggested as a potential agent for colon cancer. This study was aimed to identify the bioactive components and potential mechanisms of ginger for colon cancer prevention by an integrated network pharmacology approach.

Methods: The putative ingredients of ginger and its related targets were discerned from the TCMSP and Swiss target prediction database. After that, the targets interacting with colon cancer were collected using Genecards, OMIM, and Drugbank databases. KEGG pathway and GO enrichment analysis were performed to explore the signaling pathways related to ginger for colon cancer treatments. The PPI and compound-target-disease networks were constructed using Cytoscape 3.8.1. Finally, Discovery studio software was employed to confirm the key genes and active components from ginger.
\end{abstract}

Results: Six potential active compounds, 285 interacting targets in addition to 1356 disease-related targets were collected, of which 118 intersection targets were obtained. A total of 34 key targets including PIK3CA, SRC, and TP53 were identified through PPI network analysis. These targets were mainly focused on the biological processes of phosphatidylinositol 3-kinase signaling, cellular response to oxidative stress, and cellular response to peptide hormone stimulus. The KEGG enrichment manifested that three signaling pathways were closely related to colon cancer prevention of ginger, cancer, endocrine resistance, and hepatitis B pathways. TP53, HSP90AA1, and JAK2 were viewed as the most important genes, which were validated by molecular docking simulation.

Conclusion: This study demonstrated that ginger produced preventive effects against colon cancer by regulating multi-targets and multi-pathways with multicomponents. And, the combined data provide novel insight for ginger compounds developed as new drug for anti-colon cancer.

(Continued on next page)

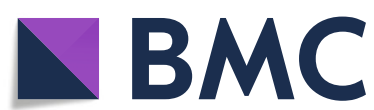

(c) The Author(s). 2021 Open Access This article is licensed under a Creative Commons Attribution 4.0 International License, which permits use, sharing, adaptation, distribution and reproduction in any medium or format, as long as you give appropriate credit to the original author(s) and the source, provide a link to the Creative Commons licence, and indicate if changes were made. The images or other third party material in this article are included in the article's Creative Commons licence, unless indicated otherwise in a credit line to the material. If material is not included in the article's Creative Commons licence and your intended use is not permitted by statutory regulation or exceeds the permitted use, you will need to obtain permission directly from the copyright holder. To view a copy of this licence, visit http://creativecommons.org/licenses/by/4.0/. The Creative Commons Public Domain Dedication waiver (http://creativecommons.org/publicdomain/zero/1.0/) applies to the data made available in this article, unless otherwise stated in a credit line to the data. 
(Continued from previous page)

Keywords: Ginger, Colon cancer, GO enrichment, KEGG enrichment, Molecular docking, 1-Monolinolein

\section{Introduction}

With the rapid development of human society and the improvement of life standard, cancer as a growing threat, is the second leading noncommunicable disease of death globally next only to cardiovascular disease [1]. Colorectal cancer is a broad term that includes patients with colon cancer and rectal cancer [2]. In 2018, there are 1,849,518 new cases of colorectum cancer worldwide and 880,792 deaths (https://gco.iarc.fr/). Currently, increasing therapeutic approaches for colon cancer treatment are available, not only the conventional treatments of surgical resection and radiotherapy but also new interventional therapies like drug therapy. However, high costs of molecular targeted therapy and chemotherapy-induced adverse effects such as nausea, vomiting, and digestive tract irritation reaction always leads to treatment drop out.

Historically medicinal botanicals, as one part of complementary medicine in the USA, have provided an important resource for discovering anticancer drug agents, and more than half of currently available drugs are related to them [3]. Ginger (Zingiber officinale), belong to Zingiberaceae family, has long been used as a traditional Chinese medicine (TCM), which mainly distributed in India, China, and Nigeria [4]. Ginger rhizome as the major medicinal part contains pungent phenolic compounds, volatile oil, diarylheptanoids, and phenylalkanoids, leading to a wide range of pharmacological effects [5, 6]. Furthermore, 6-gingerol and 6-shogaol, the representative components derived from ginger, has been reported to preventing cell proliferation against colon cancer, such as Colon-26 tumors [7, 8]. However, it is unclear whether other ginger components possess the anticancer activity.

While moving ahead with computer technology, network pharmacology incorporating a series of disciplines and techniques has emerged. Network pharmacology has been successfully introduced to reveal the therapeutic mechanisms of TCM, as it in accordance with the connotation of holistic strategy of multi-components, multi-targets and multi-pathways [9-11].

In this study, network pharmacology was conducted to establish the componentstargets-pathways-disease network to investigate the potential mechanisms of ginger in colon cancer prevention. The detailed flowchart of the study design was shown in Fig. 1. Firstly, the candidate compounds and intersection genes for colon cancer were collected. Then, the protein-protein interaction (PPI) and components-targets-pathways-disease network were constructed. In addition, Gene Ontology (GO) and Kyoto Encyclopedia of Genes and Genomes (KEGG) analysis were performed. Finally, the potential bioactive components and core targets were further validated by the molecular docking simulation.

\section{Materials and methods}

\section{Active components and corresponding target collection}

Traditional Chinese medicine systems pharmacology (TCMSP, http://tcmspw.com/ tcmsp.php) database is a platform containing many compounds from herbal medicine, related protein targets as well as its pharmacokinetic properties [12]. It was used to collect the potential active components of ginger, by filtering the metrics of oral bioavailability $(\mathrm{OB}) \geq 30 \%$ and drug-likeness $(\mathrm{DL}) \geq 0.18$ [13]. DL is a qualitative property of 


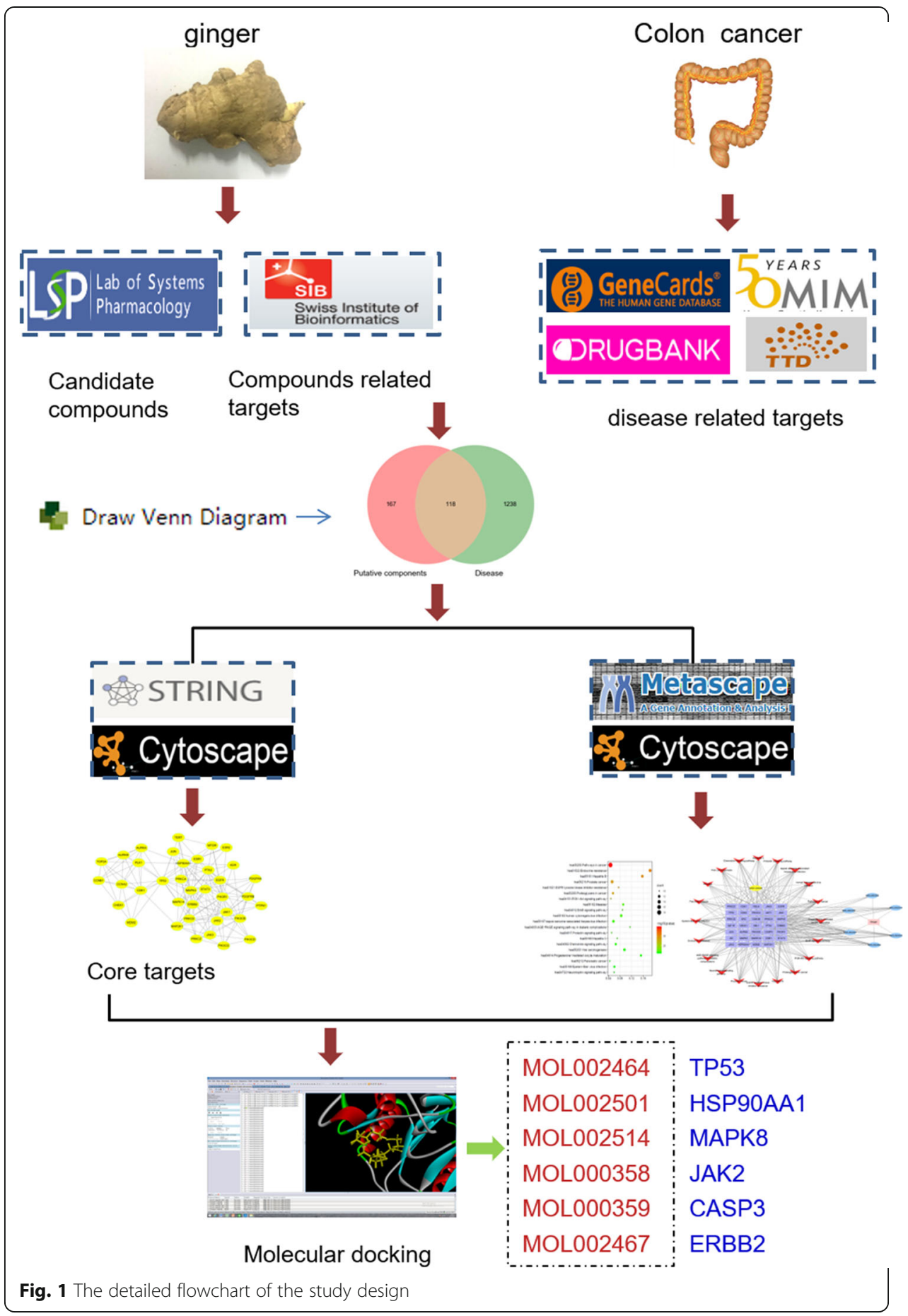

chemicals and is widely used in the early stages of drug discovery. OB refers to the relative amount and rate at which an oral drug is absorbed by the body into the systemic circulation. $\mathrm{OB}$ and DL, calculated by machine learning methods or Tanimoto coefficient, are commonly used for filtering out compounds that are unlikely to be drugs. Subsequently, protein targets interacting with those potential active compounds were predicted through TCMSP and Swiss Target Prediction (http://www. swisstargetprediction.ch/) databases [14]. 


\section{Acquisition of disease-associated targets and candidate genes}

Keywords such as "colon cancer" or "colon tumor" were used to search the known targets related to the pathogenesis of colon cancer from three major databases, GeneCards (https://www.genecards.org) [15], OMIM (https://omim.org/) [16], and Drugbank database (https://www.drugbank.ca/). To reduce the false positives, only the curated targets that directly associated with the disease were included, and the repetitive targets were removed. Then, the protein targets related to disease and ginger compounds were both imported into Uniprot database (https://www.uniprot.org/) to acquire its related gene name, gene ID and functions, respectively. The obtained genes were both inputted to draw Venn diagram (http://bioinformatics.psb.ugent.be/webtools/Venn/), and the intersection genes were collected as candidate genes.

\section{Protein-protein interaction (PPI) network}

Based on the candidate genes, a PPI network was constructed by importing the candidate genes to the Search Tool for the Retrieval of Interacting Genes (STRING, https:// string-db.org/) database, with a highest confidence of 0.9 [17]. Cytoscape software, version 3.8.1 [18] was used to visualize the PPI network. Then, three topological features, "degree", "betweenness", and "closeness" were calculated to identify the key genes. "Degree" parameter represents the number of edges associated with a node. "Betweenness" indicates the number of shortest paths between pairs of nodes, and "closeness" describes the inverse of the number of distances.

\section{GO and KEGG pathway enrichment analysis}

The Gene Ontology (GO) and Kyoto Encyclopedia of Genes and Genomes (KEGG) enrichment were performed by the Database for Metascape (https://metascape.org). GO functionally annotates key genes into three main terms, including cellular components (CCs), molecular functions (MFs), and biological processes (BPs). Besides, Cytoscape ClueGO plugin was employed to further analyze BPs enrichment. KEGG enrichment analysis unveils the possible biological process with key genes. In addition, the bubble chart of GO and KEGG enrichment analysis were performed on the bioinformatics platform (http://www.bioinformatics.com.cn/).

\section{Construction of drug-components-disease-targets-pathways network}

To characterize the therapeutic mechanisms of ginger for colon cancer, a network of drug-components-disease-targets-pathways was constructed using Cytoscape software, version 3.8.1 [18]. In the network, the nodes with different colors and shapes represent the drug, components, disease, target genes, or disease related pathways, respectively, and an "edge" is an association between the nodes.

\section{Molecular docking simulation}

A total of 8 key genes which have good correlation with other genes, active components, and pathways including SRC, PIK3R1, TP53, HSP90AA1, MAPK8, JAK2, CASP3, and ERBB2 were included in the molecular docking simulation. The PDB-ID of these target genes were accessed from Protein Data Bank (PDB) database. In brief, Discovery studio software (version 4.5.0, Biovea Inc., Omaha, NE, USA) was employed, and the 
screened active components were prepared using "Prepare Ligands" module to obtain an effective three-dismensional conformation. After removing crystallographic water molecules, the "Prepare Protein" module was used to remove the polyconformation of target protein and to supplement the incomplete amino acid residues. Subsequently, molecular docking was performed in "LibDock" module, and LibDockScore was required to evaluate affinity of the target proteins and active components. The LibDockScore of the target protein and its corresponding prototype ligand was viewed as the threshold, and the components with higher scores were regarded as the active ones for interacting with this protein.

\section{Results}

Ginger components and candidate genes associated with colon cancer

After searching, filtering, and removal of the duplicates, 6 putative components (MOL002464, MOL002501, MOL002514, MOL000358, MOL000359, and MOL002467) with $\mathrm{OB} \geq 30 \%$ and $\mathrm{DL} \geq 0.18$ were selected from TCMSP database (listed in Table 1). Besides, the representative component 6-gingerol with high contents and

Table 1 The active compounds and their properties and structures

\begin{tabular}{|c|c|c|c|c|}
\hline Mol ID & Molecule Name & OB $\%$ & DL & Structure \\
\hline MOL002464 & 1-monolinolein & 37.18 & 0.30 & \\
\hline MOL002501 & $\begin{array}{l}\text { [(1S)-3-[(E)-but-2-enyl]-2-methyl } \\
\text {-4-oxo-1-cyclopent-2-enyl](1R,3R } \\
\text { )-3-[(E)-3-methoxy-2-methyl-3-ox } \\
\text { oprop-1-enyl]-2,2-dimethylcyclop } \\
\text { ropane-1-carboxylate }\end{array}$ & 62.52 & 0.31 & \\
\hline MOL002514 & sexangularetin & 62.86 & 0.30 & \\
\hline MOL000358 & beta-sitosterol & 36.91 & 0.75 & \\
\hline MOL000359 & sitosterol & 36.91 & 0.75 & \\
\hline MOL002467 & 6-gingerol & 35.64 & 0.16 & \\
\hline
\end{tabular}


multiple pharmacological activitieswas also recruited in this study, although the DL value is 0.16 .

Meanwhile, 285 target genes in total interacting with those putative components were collected, among which 251 were obtained from the Swiss Target Prediction and 34 from TCMSP database. In addition, there remained 1356 colon cancer-associated genes, in which 1165 genes were from the GeneCards database, 156 from the OMIM, and 35 from the Drugbank. Finally, a total of 118 intersection genes, also the candidate genes, were collected for further mechanisms study of ginger on treatment of colon cancer (Fig. 2a).

\section{PPI network analysis}

The 118 candidate genes were connected to establish an initial PPI network using Cytoscape 3.8.1 that included 104 nodes and 517 edges, and 14 isolated targets genes were removed (Fig. 2b). In addition, the top two protein-protein interacting clusters were constructed (Fig. 2c and d). Genes PIK3R1, CCNA2, and TP53 were as the core targets for one cluster (37 nodes and 179 edges), while another cluster (17 nodes and 42 edges) with STAT 3 and JAK2 as the core targets. Based on the analysis of this network, only the genes with higher values of "degree", "betweenness" and "closeness" (above the median value) were collected as the key targets of ginger for colon cancer. Ultimately, 34 key targets, with PIK3CA, SRC, and TP53 as the top ones, were collected for pathway enrichment analysis (Table 2).

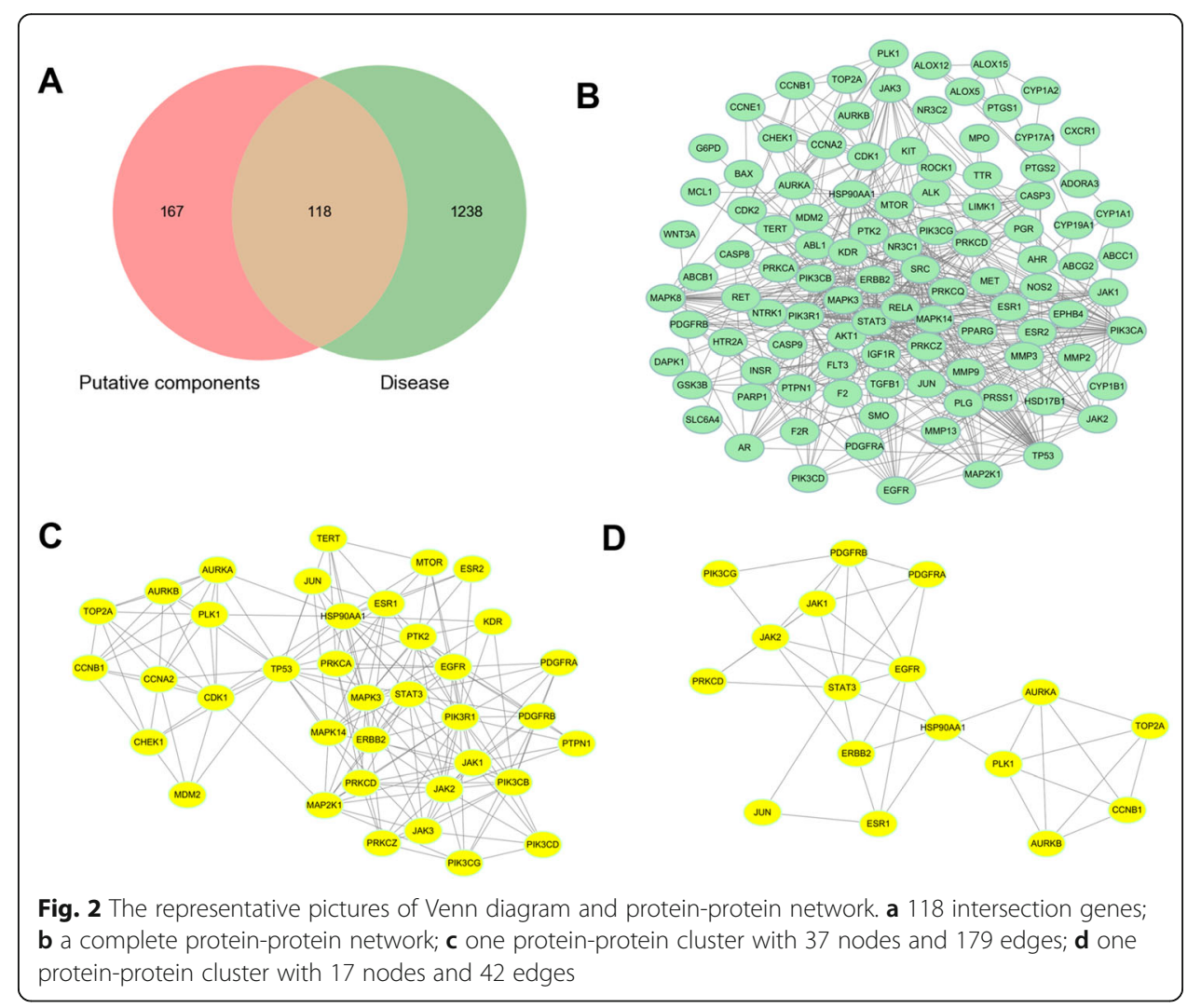


Table 2 The major targets of ginger for colon cancer treatment and the topological parameters

\begin{tabular}{|c|c|c|c|c|c|}
\hline $\begin{array}{l}\text { Uniprot } \\
\text { ID }\end{array}$ & $\begin{array}{l}\text { Gene } \\
\text { symbol }\end{array}$ & Target name & Degree & Betweenness & Closeness \\
\hline P42336 & PIK3CA & $\begin{array}{l}\text { Phosphatidylinositol 4,5-bisphosphate 3-kinase } \\
\text { catalytic subunit alpha isoform }\end{array}$ & 42 & 0.187322 & 0.572254 \\
\hline P12931 & SRC & Proto-oncogene tyrosine-protein kinase & 38 & 0.107991 & 0.568966 \\
\hline P27986 & PIK3R1 & $\begin{array}{l}\text { Phosphatidylinositol 3-kinase regulatory subunit } \\
\text { alpha }\end{array}$ & 38 & 0.106891 & 0.568966 \\
\hline P04637 & TP53 & Cellular tumor antigen p53 & 35 & 0.103695 & 0.546961 \\
\hline P27361 & MAPK3 & Mitogen-activated protein kinase 3 & 33 & 0.084312 & 0.543956 \\
\hline P40763 & STAT3 & Signal transducer and activator of transcription 3 & 31 & 0.080670 & 0.535135 \\
\hline P31749 & AKT1 & RAC-alpha serine/threonine-protein kinase & 29 & 0.068649 & 0.532258 \\
\hline P07900 & HSP90AA1 & Heat shock protein HSP 90-alpha & 27 & 0.066522 & 0.529412 \\
\hline P45983 & MAPK8 & Mitogen-activated protein kinase 8 & 23 & 0.065784 & 0.502538 \\
\hline O60674 & JAK2 & Tyrosine-protein kinase JAK2 & 20 & 0.056021 & 0.497487 \\
\hline P42338 & PIK3CB & $\begin{array}{l}\text { Phosphatidylinositol 4,5-bisphosphate 3-kinase } \\
\text { catalytic subunit beta isoform }\end{array}$ & 19 & 0.036367 & 0.492537 \\
\hline P00533 & EGFR & Epidermal growth factor receptor & 19 & 0.028652 & 0.485294 \\
\hline Q05513 & PRKCZ & Protein kinase $\mathrm{C}$ zeta type & 18 & 0.021649 & 0.480583 \\
\hline Q05655 & PRKCD & Protein kinase $C$ delta type & 18 & 0.021286 & 0.478261 \\
\hline P23458 & JAK1 & Tyrosine-protein kinase JAK1 & 18 & 0.017349 & 0.478261 \\
\hline P03372 & ESR1 & Estrogen receptor & 18 & 0.016778 & 0.473684 \\
\hline Q04206 & RELA & Transcription factor p65 & 17 & 0.014046 & 0.469194 \\
\hline Q16539 & MAPK14 & Mitogen-activated protein kinase 14 & 17 & 0.011251 & 0.469194 \\
\hline Q05397 & PTK2 & Focal adhesion kinase 1 & 15 & 0.009972 & 0.464789 \\
\hline Q02750 & MAP $2 \mathrm{~K} 1$ & $\begin{array}{l}\text { Dual specificity mitogen-activated protein kinase } \\
\text { kinase } 1\end{array}$ & 15 & 0.009205 & 0.464789 \\
\hline P04626 & ERBB2 & Receptor tyrosine-protein kinase erbB-2 & 15 & 0.008965 & 0.464789 \\
\hline P42574 & CASP3 & Caspase-3 & 15 & 0.008693 & 0.462617 \\
\hline P04150 & NR3C1 & Glucocorticoid receptor & 14 & 0.00863 & 0.460465 \\
\hline P05412 & JUN & Transcription factor AP-1 & 14 & 0.008501 & 0.458333 \\
\hline P52333 & JAK3 & Tyrosine-protein kinase JAK3 & 14 & 0.008096 & 0.450000 \\
\hline P06493 & CDK1 & Cyclin-dependent kinase 1 & 14 & 0.007042 & 0.450000 \\
\hline P17252 & PRKCA & Protein kinase $\mathrm{C}$ alpha type & 13 & 0.006885 & 0.447964 \\
\hline P10275 & $A R$ & Androgen receptor & 13 & 0.005944 & 0.445946 \\
\hline P08069 & IGF1R & Insulin-like growth factor I receptor & 11 & 0.005841 & 0.443946 \\
\hline O14965 & AURKA & Aurora kinase A & 11 & 0.005668 & 0.440000 \\
\hline P00519 & ABL1 & Tyrosine-protein kinase ABL1 & 11 & 0.005616 & 0.432314 \\
\hline Q00987 & MDM2 & E3 ubiquitin-protein ligase Mdm2 & 10 & 0.00552 & 0.415966 \\
\hline P24941 & CDK2 & Cyclin-dependent kinase 2 & 10 & 0.004538 & 0.415966 \\
\hline P49841 & GSK3B & Glycogen synthase kinase-3 beta & 8 & 0.004458 & 0.415966 \\
\hline
\end{tabular}

\section{GO enrichment analysis}

To investigate the biological function of target genes of ginger for colon cancer, the biological process was obtained by the Metascape database. As shown in Fig. 3a, 20 markedly enriched BPs terms were shown $(p<0.01)$, with transmembrane receptor protein tyrosine kinase GO: 0007169, cellular response to nitrogen compound GO: 1901699, and peptidyl-serine phosphorylation GO: 0018105 as the top ones. The size of 
the dot in bubble chart indicates the number of target genes in the corresponding function pathway, and the enrichment expresses the ratio of the number of target genes belonging to all the annotated genes located in the pathway. Besides, further analysis of the network was performed using Cytoscape plugin ClueGO, and we found that these BPs terms were mainly associated with the phosphatidylinositol 3-kinase signaling, cellular response to oxidative stress, cellular response to peptide hormone stimulus, and peptidyl-serine phosphorylation (Fig. 3b).

For CCs, the items with significant enrichment were in perinuclear region of cytoplasm GO: 0048471, transferase complex, transferring phosphorus-containing groups GO: 0061695, and microtubule organizing center GO: 0005815; and for MFs in phosphotransferase activity, alcohol group receptor GO: 0016773, kinase binding GO: 0019900, and protein tyrosine kinase activity GO: 0004713.

\section{KEGG pathway enrichment analysis}

The KEGG enrichment showed how ginger acts on the pathway, thereby playing a therapeutic role in colon cancer. Here, based on the 34 core target genes, 20 significant signaling pathways (Fig. 4) with $p<0.01$ were picked out for further analysis, including pathways in cancer (hsa05200), endocrine resistance (hsa01522), EGFR tyrosine kinase inhibitor resistance (hsa01521), and PI3K-Akt signaling pathway (hsa04151) as the top

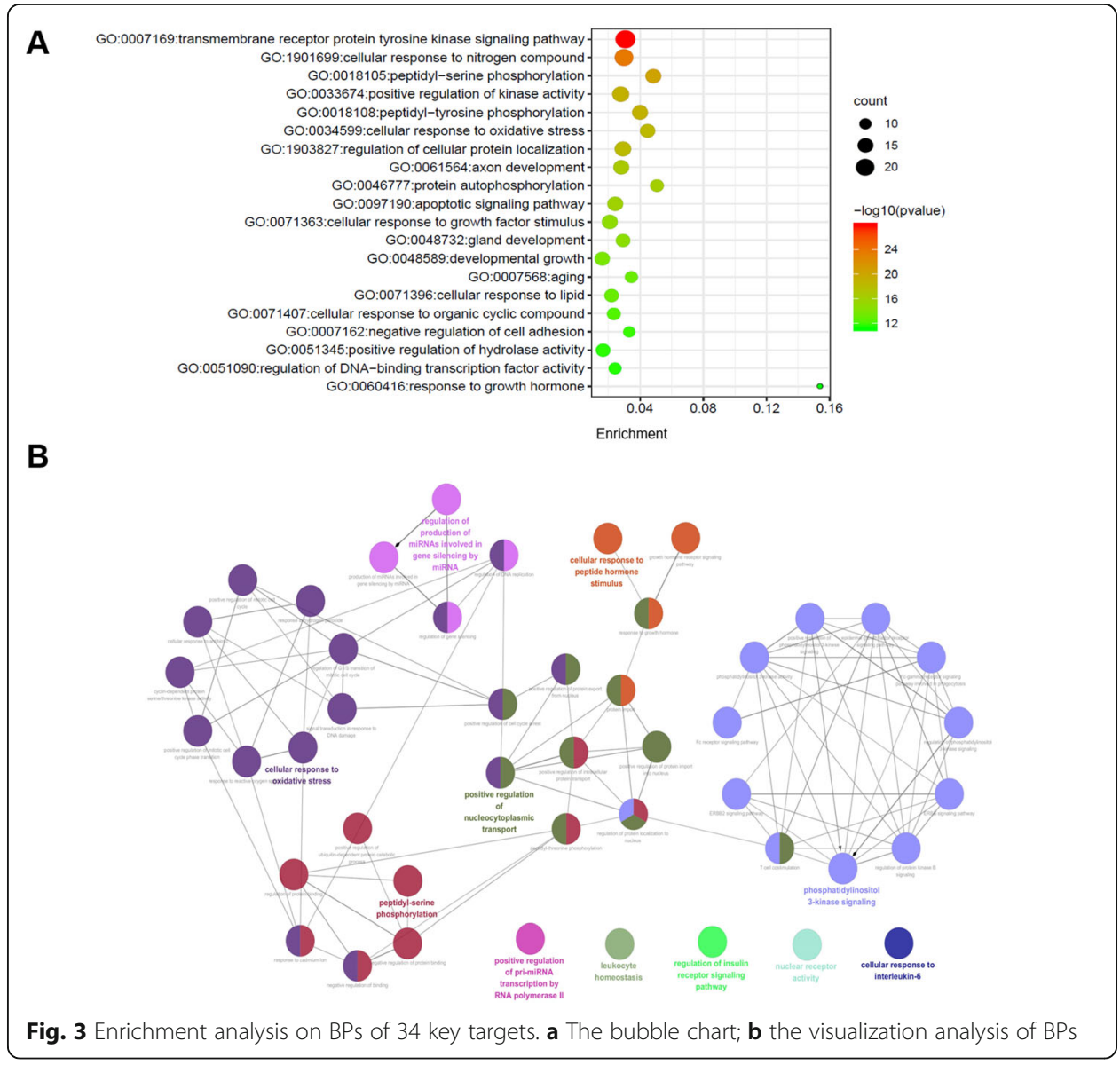




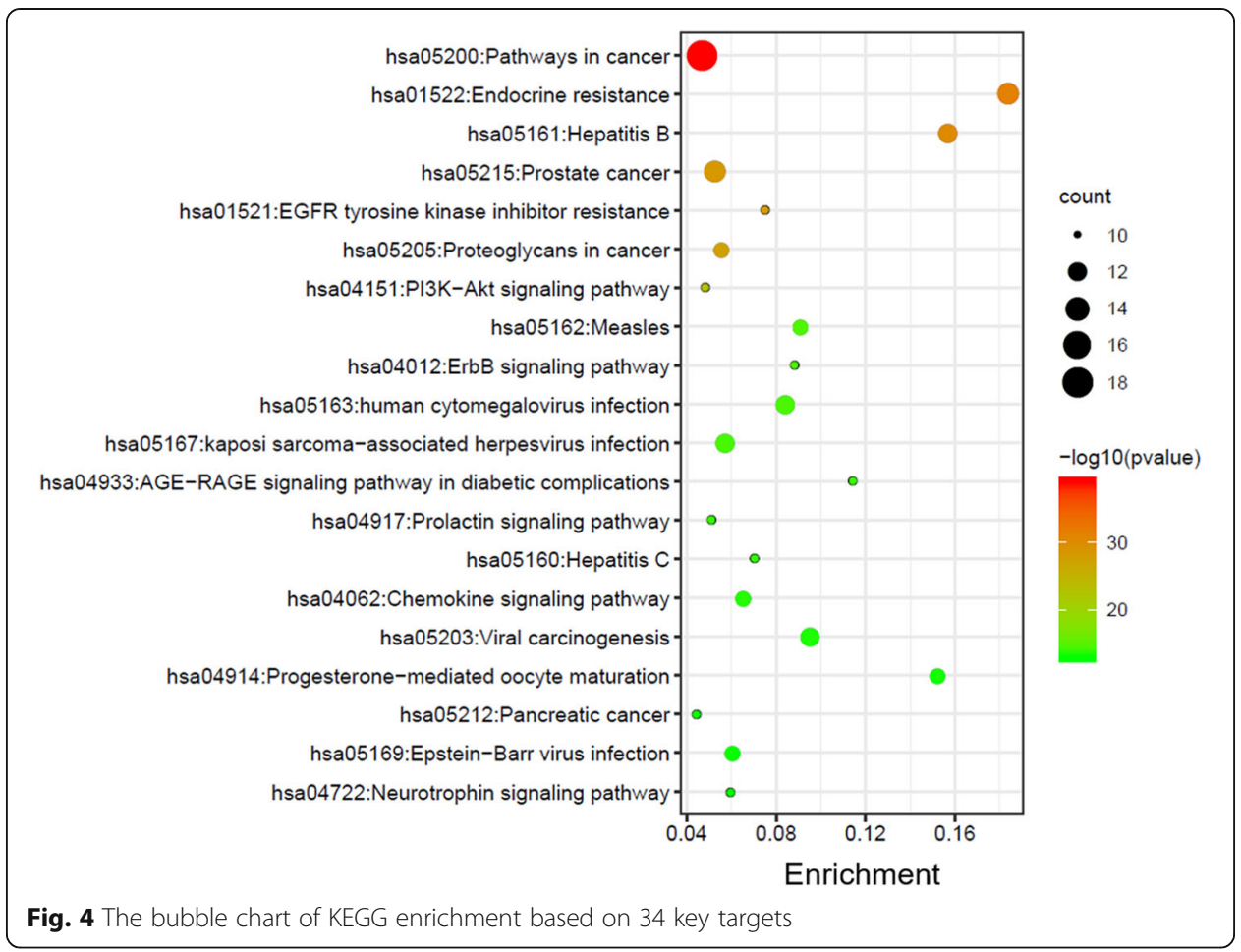

ones. Among these pathways, pathways in cancer was identified as a set of important key pathway with the most target enrichment and lowest $p$ value.

\section{Drug-components-targets-pathways-disese network}

The drug-components-targets-pathways-disease network was shown in Fig. 5, which included 62 nodes ( 6 compounds, 34 targets, and 20 pathways) and 311 edges. The pink rectangle node is the Chinese herbal medicine ginger; yellow nodes is colon cancer, $\mathrm{V}$ nodes is 20 significant signaling pathway; purple rectangle nodes are 34 key targets; blue ellipse node represents 6 potential active components, while lines represent the interactions between them. According to the network analysis, multiple components from ginger acts on at least one target genes, and 6-gingerol was regarded as the most effective compound that interacts with 17 target genes. Besides, most of target genes were regulated by at least 2 active components, and at least 10 genes potentially involved in each pathway related to colon cancer. This network analysis indicated the characteristics of multiple components and multiple targets of ginger in the treatment of colon cancer.

\section{Molecular docking results and analysis}

A total of 8 target genes which showed strong interactions with other targets, pathway and potential components were selected to binding with the 6 putative components. The binding affinities of the testing components, indicating as LibDock Scores, were compared to the original ligands of target protein. As LibDock Scores displayed in Table 3 and heat map of the docking score exhibited in Fig. 6, all the testing components had strong interactions than the prototype ligands, or similar effects to the 


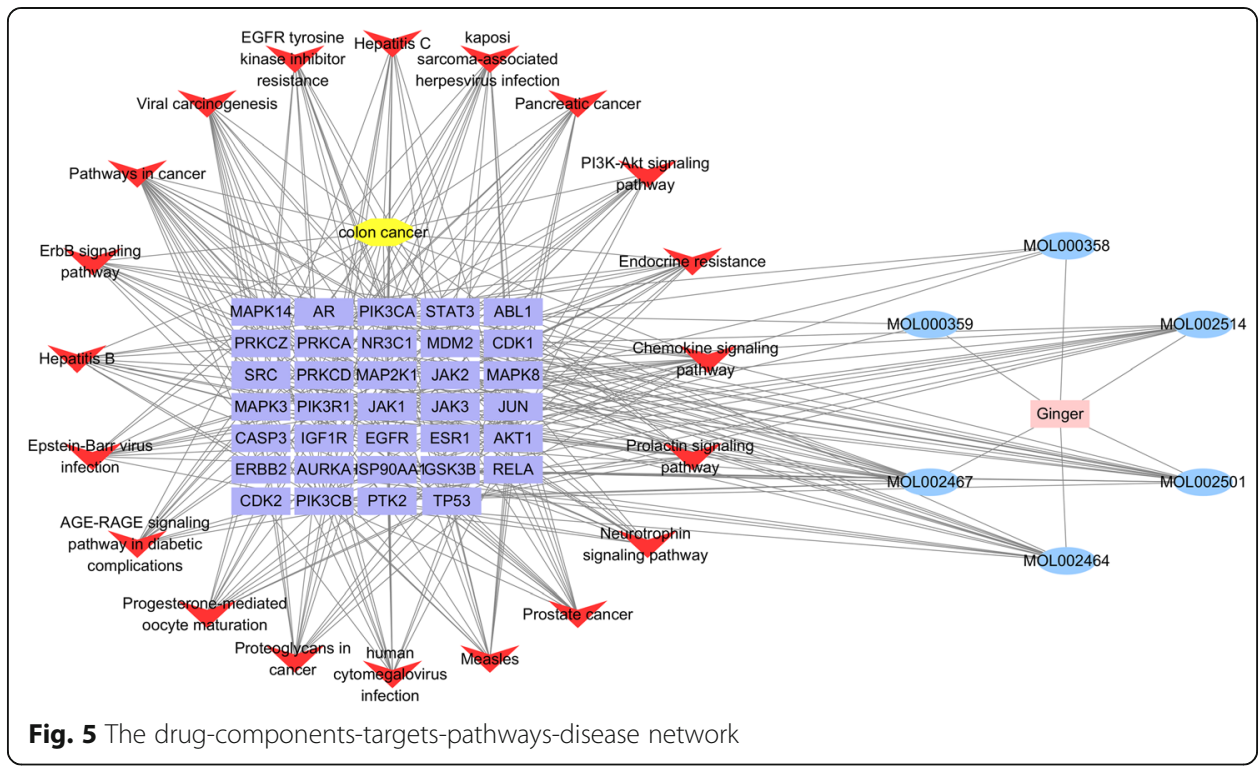

ligands, with TP53, HSP90AA1 and JAK2. Especially for compound 1-monolinolein (MOL002464) showed good binding affinities with all the tested targets, except for SRC. In addition, 6-gingerol also showed a strong interaction with targets JAK2 and CASP3, while beta-sitosterol (MOL000358) and sitosterol (MOL000359) with ERBB2. These findings revealed that the six components including MOL002464, MOL002501, MOL002467, MOL000358, MOL002514, and MOL000359 were forecasted as the active components of ginger for colon cancer and TP53, HSP90AA1, and JAK2 were the major target for reaching this effect. The representative molecular docking results of the major targets and active components of ginger were exhibited in Fig. 7.

\section{Discussion}

Cancer is a worldwide problem, especially for the prevention of postoperative spread. Chinese herbal medicine, as the characteristics of safety and minimal side effects, are increasingly used as cancer-preventive agents for patients who prefer a healthy treatment after tumor resection. Recently, the network pharmacology has become an advanced method to analyze the complex mechanisms and collect active ingredients of Chinese herbs or TCM formula. This study indicated that ginger possess the effect of preventing colon cancer by regulating multi-targets with multi-components.

Specially, six ingredients of ginger were screened out. In which, 6-gingerol as a naturally occurring plant phenol, is the most abundant component in fresh ginger. It has been shown that 6-gingerol play an anti-colorectal cancer effect by inhibiting leukotriene A (4) hydrolase expression and induction of G2/M arrest [19-21]. It is worth noting that this compound exhibited compact relationships with 17 key target genes, indicating the potential of 6-gingerol as a leading compound in colon cancer prevention. However, the LibDock Scores of 6-gingerol is lower than 1-monolinolein, which may be due to the poor DL property $(\mathrm{DL}<0.18)$. Therefore, structural variation should be applied for improving its pharmacokinetics characteristics to reach a reasonable drug-like property. Other active components, like beta-sitosterol, a main dietary 
Table 3 The LibDock Scores of 8 core targets and their interacting compounds

\begin{tabular}{|c|c|c|c|}
\hline Target & PDB ID & Components & LibDock Score \\
\hline \multirow[t]{7}{*}{$\overline{S R C}$} & $2 \mathrm{BDF}$ & Ligand 1 & 134.987 \\
\hline & & MOL002464 & 125.359 \\
\hline & & MOL002501 & 112.922 \\
\hline & & MOL002467 & 115.549 \\
\hline & & MOL000358 & 116.823 \\
\hline & & MOL002514 & 106.056 \\
\hline & & MOL000359 & 116.823 \\
\hline \multirow[t]{7}{*}{ PIK3R1 } & $4 Z O P$ & Ligand 2 & 124.433 \\
\hline & & MOL002464 & 127.529 \\
\hline & & MOL002501 & 118.423 \\
\hline & & MOL002467 & 112.848 \\
\hline & & MOL000358 & 122.996 \\
\hline & & MOL002514 & 107.208 \\
\hline & & MOL000359 & 122.996 \\
\hline \multirow[t]{7}{*}{ TP53 } & $501 F$ & Ligand 3 & 104.359 \\
\hline & & MOL002464 & 146.759 \\
\hline & & MOL002501 & 127.538 \\
\hline & & MOL002467 & 128.495 \\
\hline & & MOL000358 & 129.767 \\
\hline & & MOL002514 & 125.236 \\
\hline & & MOL000359 & 133.846 \\
\hline \multirow[t]{7}{*}{ HSP90AA1 } & $4 \mathrm{BQG}$ & Ligand 4 & 93.3973 \\
\hline & & MOL002464 & 137.279 \\
\hline & & MOL002501 & 130.015 \\
\hline & & MOL002467 & 123.039 \\
\hline & & MOL000358 & 124.673 \\
\hline & & MOL002514 & 117.016 \\
\hline & & MOL000359 & 124.673 \\
\hline \multirow[t]{7}{*}{ MAPK8 } & $4 \mathrm{E} 73$ & Ligand 5 & 118.446 \\
\hline & & MOL002464 & 126.778 \\
\hline & & MOL002501 & 105.652 \\
\hline & & MOL002467 & 99.7431 \\
\hline & & MOL000358 & 110.918 \\
\hline & & MOL002514 & 95.7262 \\
\hline & & MOL000359 & 110.918 \\
\hline \multirow[t]{7}{*}{ JAK2 } & $3 \mathrm{KCK}$ & Ligand 6 & 102.423 \\
\hline & & MOL002464 & 116.567 \\
\hline & & MOL002501 & 105.396 \\
\hline & & MOL002467 & 104.779 \\
\hline & & MOL000358 & 109.404 \\
\hline & & MOL002514 & 106.601 \\
\hline & & MOL000359 & 109.106 \\
\hline \multirow[t]{2}{*}{ CASP3 } & 1RE1 & Ligand 7 & 96.0197 \\
\hline & & MOL002464 & 115.813 \\
\hline
\end{tabular}


Table 3 The LibDock Scores of 8 core targets and their interacting compounds (Continued)

\begin{tabular}{|c|c|c|c|}
\hline Target & PDB ID & Components & LibDock Score \\
\hline & & MOL002501 & 100.826 \\
\hline & & MOL002467 & 105.848 \\
\hline & & MOL000358 & 82.4267 \\
\hline & & MOL002514 & 104.926 \\
\hline & & MOL000359 & 84.5633 \\
\hline \multirow[t]{7}{*}{ ERBB2 } & 3РPO & Ligand 8 & 120.872 \\
\hline & & MOL002464 & 148.403 \\
\hline & & MOL002501 & 109.008 \\
\hline & & MOL002467 & 94.1141 \\
\hline & & MOL000358 & 139.536 \\
\hline & & MOL002514 & 91.1141 \\
\hline & & MOL000359 & 139.034 \\
\hline
\end{tabular}

phytosterol in plants, was found to be preventive on the growth of HT116 human colon cancer cells and this function was associated with induction of Bax and activation of Caspases [22]. This above evidence indicates the activities of these ingredients in ginger for treatment of colon cancer, however, further clinical experiment is still needed, especially for the most active component of 1-monolinolein.

By target fishing, a total of 34 key genes were screened as the most important ones that contribute to the colon cancer prevention of ginger. These potential target genes were placed in the Metascape for KEGG pathway analysis, and 20 significant pathways that may be regulated by ginger in the treatment of colon cancer were identified, including pathways in cancer, endocrine resistance, hepatitis B, PI3K-Akt signaling pathway, and EGFR tyrosine kinase inhibitor resistance. Importantly, PI3K-Akt pathway is a

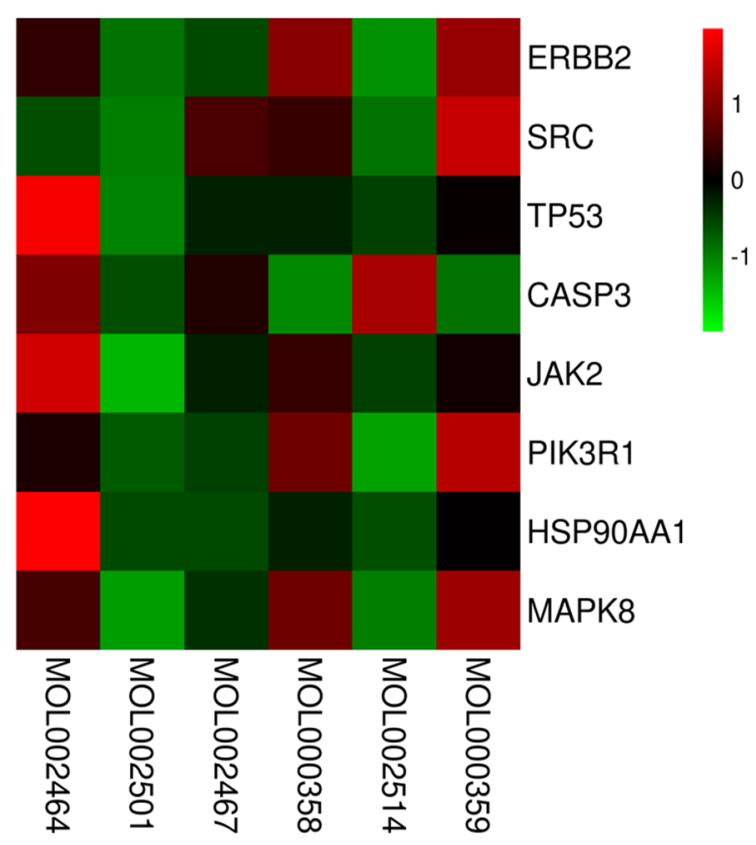

Fig. 6 The heat map of the docking score 


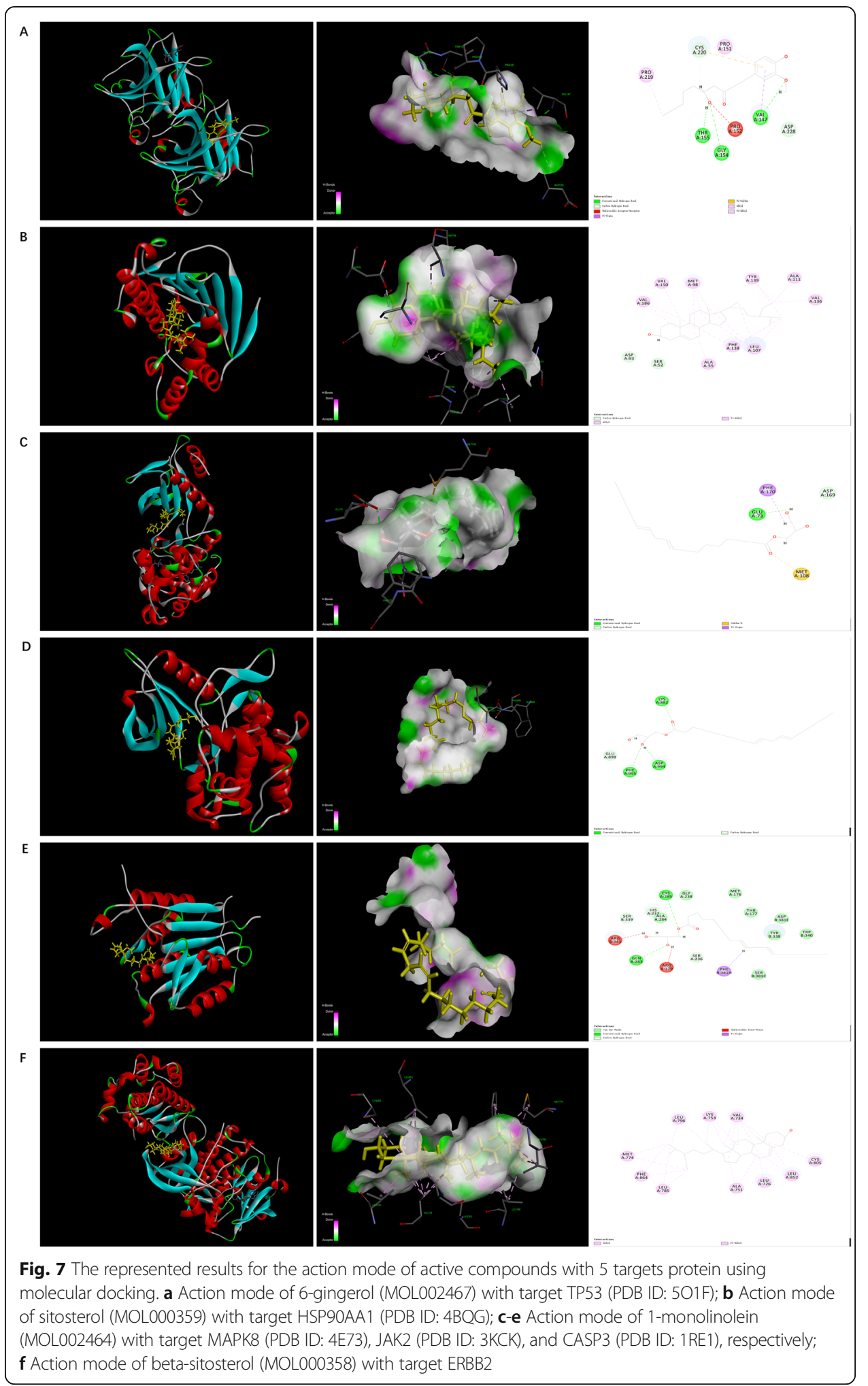


critical signal cascade focusing on serine/threonine kinase Akt, and AKT1, PIK3CA, MAPK3, TP53 genes were involved in this pathway. It perhaps the most commonly activated signaling pathway in human cancer and misregulation of the PI3K-Akt pathway has been revealed to be closely associated with pathogenetic process of colon cancer [23, 24]. By analyzing GO enrichment, we found that phosphatidylinositol 3-kinase signaling is an important biological process of ginger on regulating cancer-related pathways, which may be contribute to the regulation of PI3K-Akt pathway in this study. Another signaling pathway of EGFR tyrosine kinase inhibitor have been effectively used as a promising target for clinical treatment of non-squamous non-small cell lung cancer [25, 26]. However, some of responders relapsed because of acquired resistance. Recent studies showed that combination of EGFR tyrosine kinase inhibitor and AKT inhibitors could be a rational therapeutic approach for lung cancer patients [27]. Therefore, PI3K-Akt signaling pathway and EGFR tyrosine kinase inhibitor resistance pathways can be considered as the significant pathways of ginger for treating colon cancer. Besides, some biological process, such as cellular response to oxidative stress, has been found to take part in regulating signaling pathways related to human colon cancer [28, 29]. Reactive oxygen species (ROS) is an umbrella term for an array of derivatives of oxygen, and elevated level of ROS resulted in molecular damage, denoted as "oxidative distress" [30]. It is increasingly clear that reactive oxygen species (ROS) including superoxide and hydrogen peroxide, positioned at a crossroads potentially linking the cancer and immune microenvironment [31]. Further study demonstrated that targeting mitochondrial complex I with metformin led to reduction of complex I generated ROS and reduced tumorigenesis in xenograft mouse models [32].

According to the compound-target-pathway-disease interaction network, each active component acts on at least one target genes and most of the target acts on at least one pathway. For example, TP53 as a tumor suppressor gene was found to be involved in 11 pathways, such as EGFR tyrosine kinase inhibitor resistance and PI3K-Akt signaling pathway. Another important gene of PIK3R1 was associated with all the significant pathways. Furthermore, the core targets TP53, HSP90AA1, and JAK2 were verified to be the potential targets of ginger for treating colon cancer. These findings revealed that the anti-cancer effect of ginger was played through regulating multi-targets by multi-components. However, the illustration in the molecular levels of ginger prevented colon cancer need to be provided in the future. Meanwhile, another limitation of this investigation is that it was focused on lipid substances of ginger, and large molecular compounds such as polysaccharides and proteins were not included. Further research is required to supplement this data.

\section{Conclusion}

Collectively, 6 active components were identified from ginger and 285 targets in addition to 1356 disease-related targets in the treatment of colon cancer were collected. In the String analysis, a total of 34 candidate targets were yielded, and 10 signaling pathways including pathways in cancer, PI3K-Akt signaling pathway, and EGFR tyrosine kinase inhibitor resistance were observed to play an important role in the mechanism of ginger for colon cancer prevention. In addition, TP53, HSP90AA1, and JAK2 were speculated to be the most important target proteins. These results might be promising to searching for leading compounds and the developments of new drug for colon cancer, however, continued experiments are demanded to available the findings. 


\section{Acknowledgements}

The authors wish to thank the editor and the anonymous reviewers whose constructive comments are very helpful in strengthening the presentation of this paper. This work was financially supported by the "Xinglin scholars" talent research promotion project (NO. QNXZ2019025 \& NO. CXTD2018007) of Chengdu University of TCM.

\section{Authors' contributions}

ZMM, AL and WCJ: study design; acquisition of data; analysis of data; drafting of the manuscript. WD, LF, and ZR: acquisition of data; critical revision of the manuscript. YX and $\mathrm{HL}$ : revision of the manuscript and study supervision. All the author(s) read and approved the final manuscript.

\section{Availability of data and materials}

All data are available in the manuscript and they are exhibited in figures and tables.

\section{Ethics approval and consent to participate}

No applicable.

\section{Competing interests}

Authors declare no conflict of interest.

Received: 25 June 2020 Accepted: 14 December 2020

Published online: 11 January 2021

\section{References}

1. Jemal A, Bray F, Center MM, et al. Global cancer 536 statistics. CA Cancer J Clin. 2011:61:69-90.

2. Nasir A, Bullo MMH, Ahmed Z, et al. Nutrigenomics: epigenetics and cancer prevention: a comprehensive review. Crit Rev Food Nutr. 2020:60(8):1375-87.

3. Gordaliza M. Natural products as leads to anticancer drugs. Clin Transl Oncol. 2007:9:767-76.

4. Govindarajan VS. Ginger: chemistry, technology, and quality evaluation: part 1. Crit Rev Food Sci. 1982;17:1-96.

5. Guo T, Tan SB, Wang Y, et al. Two new monoterpenoid glycosides from the fresh rhizome of Tongling white ginger (Zingiber officinale). Nat Prod Res. 2018;32:71-6.

6. Kubra IR, Mohan LJ. An impression on current developments in the technology, chemistry, and biological activities of ginger (Zingiber officinale roscoe). Crit Rev Food Technol. 2012;52:651-88.

7. Liao DW, Cheng C, Liu JP, et al. Characterization and antitumor activities of polysaccharides obtained from ginger (Zingiber officinale) by different extraction methods. Int J Biol Macromol. 2020;152:894-903.

8. Zhang MZ, Xiao B, Wang $\mathrm{H}$, et al. Edible ginger-derived Nano-lipids loaded with doxorubicin as a novel drug-delivery approach for colon cancer therapy. Mol Ther. 2016;24:1783-96.

9. Song $X Q$, Zhang $Y$, Dai EQ, et al. Prediction of triptolide targets in rheumatoid arthritis using network pharmacology and molecular docking. Int Immunopharmacol. 2020;80:106179.

10. Kim SK, Lee S, Lee MK, et al. A systems pharmacology approach to investigate the mechanism of Oryeong-sanformula for the treatment of hypertension. J Ethnopharmacol. 2019;244:112-29.

11. Liu SY, Wu JR, Zhu YL, et al. Network pharmacology-based approach to investigate the mechanisms of Shenqi Fuzheng injection in the treatment of breast cancer. Eur J Integr Med. 2020;34:101064.

12. Ru JL, Li P, Wang JN, et al. TCMSP: a database of systems pharmacology for drug discovery from herbal medicines. J Cheminf. 2014:6:13.

13. Qian C, Zhang YL, Ma YH, et al. A network pharmacology approach to investigate the mechanism of Shuxuening injection in the treatment of ischemic stroke. J Ethnopharmacol. 2020;257:112891.

14. Gfeller D, Grosdidier A, Wirth M, et al. SwissTargetPredicition: a web server for target predicition of bioactive small molecules. Nucleic Acids Res. 2014:42:W32-8 Web Server issue

15. Stelzer G, Dalah I, Stein Tl, et al. In-silico human genomics with GeneCards. Hum Genom. 2011;5(6):709-17.

16. Amberger JS, Bocchini CA, Schiettecatte F, et al. OMIM.org: online mendelian inheritance in man, an online catalog of human genes and genetic disorders. Nucleic Acids Res. 2015;43:D789-98.

17. Szklarczyk D, Franceschini A, Kuhn M, et al. STRING v11: protein-protein association networks with increased coverage, supporting functional discovery in genome-wide experimental datasets. Nucleic Acids Res. 2019:47:D607-13.

18. Kohl M, Wiese S, Warscheid B. Cytoscape: software for visualization and analysis of biological networks (M)//data mining in proteomics. Methods Mol Biol. 2011;96:291-303.

19. Kumara M, Shylajab MR, Nazeemc PA, et al. 6-Gingerol is the most potent anticancerous compound in ginger (Zingiber officinale Rosc.). J Develop Drugs. 2017;6:1-10.

20. Jeong $\mathrm{CH}$, Bode AM, Pugliese A, et al. [6]-Gingerol suppresses colon cancer growth by targeting leukotriene A4 hydrolase. Cancer Res. 2009:69(13):5584-91.

21. Lin CB, Lin CC, Gregory JT. 6-Gingerol inhibits growth of colon cancer cell LoVo via induction of G2/M arrest. Evid Based Complement Alternat Med. 2012;2012:326096.

22. Choi YH, Kong KR, Kim YA, et al. Induction of Bax and activation of Caspases during beta-sitosterol-mediated apoptosis in human colon cancer cells. Int J Oncol. 2003;23(6):1657-62.

23. Wang LY, Bo XT, Yi XY, et al. Exosome-transferred LINC01559 promotes the progression of gastric cancer via PI3K/AKT signaling pathway. Cell Death Dis. 2020;11(9):723.

24. Li XF, Tian RF, Liu L, et al. Andrographolide enhanced radiosensitivity by downregulating glycolysis via the inhibition of the PI3K-Akt-mTOR signaling pathway in HCT116 colorectal cancer cells. J Int Med Res. 2020;48(8):1-17.

25. Chen Y, Wu J, Yan HF, et al. Lymecycline reverses acquired EGFR-TKI resistance in non-small-cell lung cancer by targeting GRB2. Pharmacol Res. 2020;159:105007. 
26. Shanmugapriya K, Kim H, Kang HW. Epidermal growth factor receptor conjugated fucoidan/alginates loaded hydrogel for activating EGFR/AKT signaling pathways in colon cancer cells during targeted photodynamic therapy. Int I Biol Macromol. 2020;158:1163-74.

27. Chen C, Liu WR, Zhang B, et al. LncRNA H19 downregulation confers erlotinib resistance through upregulation of PKM2 and phosphorylation of AKT in EGFR-mutant lung cancers. Cancer Lett. 2020;486:58-70.

28. Sruthi S, Nageswaran S. Curcumin induced apoptosis is mediated through oxidative stress in mutated p53 and wild type p53 colon adenocarcinoma cell lines. J Biochem Mol Toxicol. 2020;34:e22616.

29. Ozbolat SN, Ayna A. Chrysin suppresses HT-29 cell death induced by diclofenac through apoptosis and oxidative damage. Nutr Cancer J. 2020;1801775.

30. Sies H, Jones DP. Reactive oxygen species (ROS) as pleiotropic physiological signalling agents. Nat Rev Mol Cell Biol. 2020;21:363-83.

31. Rhee SG. Cell signaling. $\mathrm{H}_{2} \mathrm{O}_{2}$ a necessary evil for cell signaling. Science. 2006;312:1882-3.

32. Weinberg $\mathrm{F}$, Ramnath $\mathrm{N}$, Nagrath $\mathrm{D}$. Reactive oxygen species in the tumor microenvironment: an overview. Cancers. 2019;11:1191.

\section{Publisher's Note}

Springer Nature remains neutral with regard to jurisdictional claims in published maps and institutional affiliations.

Ready to submit your research? Choose BMC and benefit from:

- fast, convenient online submission

- thorough peer review by experienced researchers in your field

- rapid publication on acceptance

- support for research data, including large and complex data types

- gold Open Access which fosters wider collaboration and increased citations

- maximum visibility for your research: over $100 \mathrm{M}$ website views per year

At $\mathrm{BMC}$, research is always in progress.

Learn more biomedcentral.com/submissions 